\title{
Carcinoma de células renales con translocación del cromosoma Xp11.2: reporte de un caso y revisión de la bibliografía
}

\author{
Renal cell carcinoma with chromosome Xp11.2 \\ translocation: A case report and literature review
}

\author{
Roberto Patricio López-Maguey, Luis Trujillo-Ortiz, Gustavo Morales-Montor, Rodrigo Pérez-Becerra, Josette \\ Staufert-Gutiérrez, Carlos Pacheco-Gahbler, Sara Parraguirre-Martínez, Haydee Martínez-Hernández, María \\ Delia Pérez Montiel Gómez
}

\begin{abstract}
Resumen
ANTECEDENTES: El carcinoma renal con translocación del cromosoma Xp11.2 fue reconocido en 2004 por la Organización Mundial de la Salud. Esta neoplasia se asocia con diversos microarreglos, principalmente del gen TFE3 en el cromosoma Xp11. La prevalencia de la translocación varía de $1-1.6 \%$ de todos los tumores renales en la población adulta.

CASO CLÍNICO: Paciente femenina de 64 años, que inició dos meses previos a su internamiento con dolor lumbar izquierdo, con irradiación al cuadrante superior ipsilateral de moderada intensidad. La urotomografía reportó un tumor en el polo inferior y en la parte media del riñón izquierdo, de $20 \times 17 \times 14 \mathrm{~cm}$, de bordes irregulares, heterogéneo, con calcificaciones; crecimiento ganglionar perihiliar bilateral, adyacente a la aorta, que desplazaba en sentido anterior a la vena cava. Los exámenes de laboratorio reportaron hemoglobina $10 \mathrm{~g} / \mathrm{dL}$ y calcio $9.2 \mathrm{mEq} / \mathrm{dL}$. Se llevó a cabo la nefrectomía radical abierta con linfadenectomía (paraórtica), donde se encontraron abundantes ganglios en el mesenterio y de manera contralateral en el hilio renal. El servicio de Patología estableció el diagnóstico de carcinoma de células renales con translocación del cromosoma Xp11.2 (inmunohistoquímica TFE-3 positivo), con metástasis a un ganglio. Actualmente se encuentra en el cuarto ciclo de quimioterapia con pazopanib, con enfermedad diseminada a los pulmones, mesenterio y la región cervical.

CONCLUSIÓN: El carcinoma de células renales con translocación del cromosoma Xp11.2 suele afectar a niños y adultos jóvenes, no provoca síntomas y las masas renales son indoloras. Puesto que la mayor parte se diagnostican en estadios avanzados, el pronóstico es desfavorable.
\end{abstract}

PALABRAS CLAVE: Tumor de translocación; carcinoma renal; metástasis.

\section{Abstract}

BACKGROUND: Renal cell carcinoma with translocation on chromosome Xp11.2 was recognized in 2004 by the World Health Organization. That neoplasia is associated with different micro-arrangements, particularly of the TFE3 gene on the Xp11 chromosome. Prevalence of the translocation varies from $1-1.6 \%$ of all kidney tumors in the adult population.

CLINICAL CASE: A 64-year-old woman presented with moderate left lumbar pain radiating to the ipsilateral upper quadrant two months prior to hospital admission. Computed tomography urogram identified a heterogeneous tumor at the lower pole and middle portion of the left kidney that measured $20 \times 17 \times 14 \mathrm{~cm}$, with irregular edges and calcifications. There was bilateral perihilar lymph node enlargement adjacent to the aorta that anteriorly displaced the vena cava. Laboratory tests reported hemoglobin of $10 \mathrm{~g} / \mathrm{dL}$ and calcium of $9.2 \mathrm{mEq} / \mathrm{dL}$. Open radical nephrectomy with para-aortic lymphadenectomy was performed, revealing abundant lymph nodes in the mesentery
División de Urología, Hospital General Dr. Manuel Gea González, Ciudad de México.

Recibido: octubre 2017

Aceptado: septiembre 2018

Correspondencia

Roberto Patricio López Maguey rpatloma@gmail.com

Este artículo debe citarse como López-Maguey RP, Trujillo-Ortiz L, Morales-Montor G, Pérez-Becerra R, Staufert-Gutiérrez J, Pacheco-Gahbler C, Parraguirre-Martínez S, MartínezHernández H, Pérez Montiel Gómez MD. Carcinoma de células renales con translocación del cromosoma Xp11.2: reporte de un caso y revisión de la bibliografía. Rev Mex Urol. 2018 septoct;78(5):389-396.

DOI: https://doi.org/10.24245/revmexurol.v78i5.1750 
and in the contralateral renal hilum. Pathology study diagnosis was renal carcinoma with Xp11.2 translocation (immunohistochemistry positive for TFE-3), with metastasis to one lymph node. The patient is currently undergoing the fourth cycle of chemotherapy with pazopanib, with disease dissemination to the lungs, mesentery, and cervical region. CONCLUSION: Renal cell carcinoma with Xp11.2 translocation usually affects children and young adults. It does not cause symptoms and the kidney masses are painless. Given that the majority of cases are diagnosed at late stages, outcome is unfavorable. KEYWORDS: Translocation tumor; Renal carcinoma; Metastasis.

\section{ANTECEDENTES}

Los carcinomas de células renales son tumores heterogéneos, relacionados con $90 \%$ de todas las neoplasias malignas del riñón en pacientes adultos. Los subtipos más comunes son: carcinoma de células claras (60-75\%), papilar (10-15\%), cromófobo $(5 \%)$ y de túbulos colectores, cada uno con cualidades moleculares y genéticas únicas. El carcinoma de células renales asociado con translocación del cromosoma Xp11.2 y fusión del gen TFE3 es un subtipo excepcional de neoplasia renal. ${ }^{1,2}$

El carcinoma de células renales con translocación del cromosoma Xp11.2 fue reconocido en 2004 por la OMS. Esta neoplasia se ha reportado en $15 \%$ de las tumoraciones renales de pacientes adultos. ${ }^{3}$ Difícilmente crece como un tumor primario, más allá de los límites normales del riñón. La mayor parte afecta a la población pediátrica y adultos jóvenes (menores de 45 años), con mayor prevalencia en mujeres; un tercio de la incidencia se registra en pacientes jóvenes y $0.2-5 \%$ en adultos. ${ }^{4,5}$

Este tumor se caracteriza por ser agresivo en pacientes de edad avanzada, con metástasis a distancia y repercusiones clínicas desfavorables cuando se diagnostica en estadios avanzados. ${ }^{1,4}$ La translocación del cromosoma Xp11.2 tiene estructura papilar o alveolar y está compuesto por células con citoplasma eosinofílico voluminoso o claro. ${ }^{6,7}$ Desde el punto de vista patológico, se origina de la médula renal, diferente del carcinoma renal de células claras típico que se forma en la corteza renal. ${ }^{4,8}$

\section{CASO CLÍNICO}

Paciente femenina de 64 años, atendida en el servicio de Urología por diagnóstico de tumor renal izquierdo. La paciente inició su cuadro clínico dos meses antes de acudir a consulta, con dolor lumbar izquierdo, con irradiación al cuadrante superior ipsilateral de moderada intensidad. Durante el interrogatorio negó tener hematuria o algún síntoma urinario adicional. La urotomografía reportó, en la fase simple, aumento de volumen en el polo inferior y en la parte media del riñón izquierdo, de aproximadamente $20 \times 17 \times 14 \mathrm{~cm}$, de bordes irregulares, densidad heterogénea e imágenes compatibles con calcificaciones, con promedio de $30 \mathrm{UH}$ (unidades Hounsfield), que se reforzaba de forma irregular en la fase contrastada, entre 90-100 UH. También se identificó aumento de volumen y densidad de estructuras compatible con crecimiento ganglionar perihiliar bilateral, adyacente a la arteria aorta, desplazando en sentido anterior a la vena cava (Figuras $\mathbf{1}$ y $\mathbf{2}$ ).

El estudio de imagen coincidió con el diagnóstico radiológico de tumor renal izquierdo de gran volumen, con aparente actividad ganglionar. La radiografía postero-anterior de tórax no demostró actividad tumoral. Los exámenes de laboratorio prequirúrgicos reportaron: hemoglobina $10 \mathrm{~g} /$ 


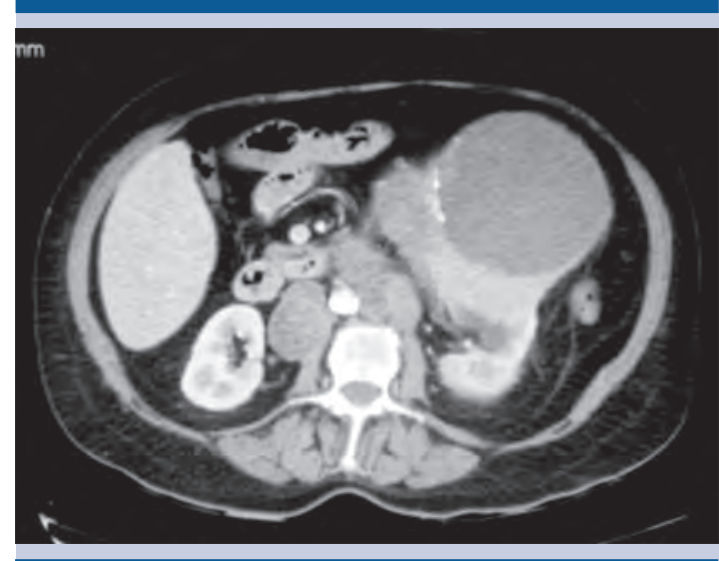

Figura 1. Tomografía de abdomen, corte axial, que muestra el riñón izquierdo con aumento de tamaño $(20 \times 17 \times 14 \mathrm{~cm})$, bordes regulares y calcificaciones.

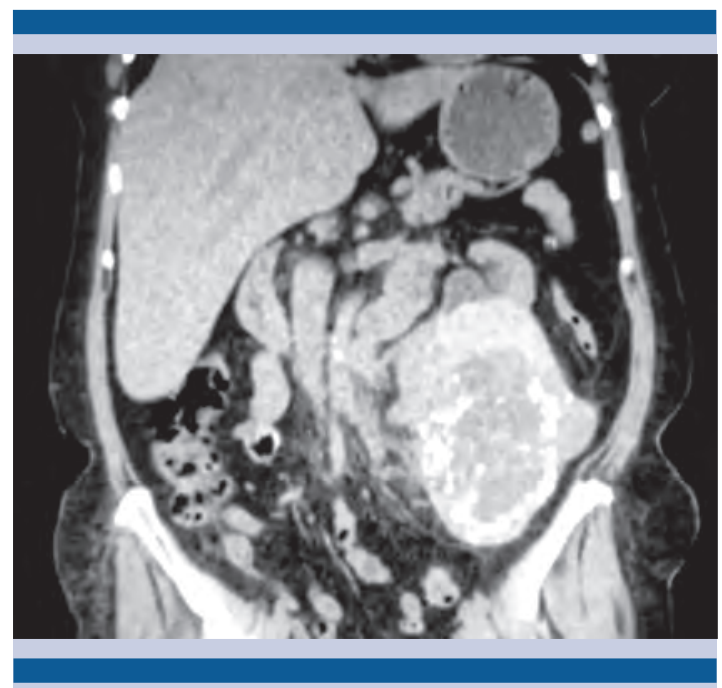

Figura 2. Tomografía, corte coronal, que evidencia una tumoración con áreas calcificadas heterogéneas, además de desplazamiento lateral de estructuras vasculares y abundantes ganglios paraórticos.

$\mathrm{dL}$, hematocrito $31.7 \%$, plaquetas $47110^{3} / \mu \mathrm{L}$, leucocitos $14.610^{3} / \mu \mathrm{L}$; glucosa $110 \mathrm{mg} / \mathrm{dL}$; BUN $18.1 \mathrm{mg} / \mathrm{dL}$, creatinina $0.7 \mathrm{mg} / \mathrm{dL}$, calcio 9.2 $\mathrm{mEq} / \mathrm{dL}$, fosforo $3.7 \mathrm{mEq} / \mathrm{dL}$, sodio $134 \mathrm{mEq} / \mathrm{L}$, potasio $4 \mathrm{mEq} / \mathrm{L}$, cloro $97 \mathrm{mEq} / \mathrm{L}$, fosfatasa alcalina $130 \mathrm{mg} / \mathrm{dL}$ y deshidrogenasa láctica $445 \mathrm{mg} /$
dL. El examen general de orina resultó negativo para hematuria e infección. Grupo sanguíneo $\mathrm{O}$ negativo. Tiempos de coagulación y pruebas de función hepática sin alteraciones.

Se llevó a cabo la nefrectomía radical izquierda abierta con linfadenectomía de ganglios paraaórticos hasta la bifurcación de la aorta; se observaron ganglios en el hilio renal contralateral, fijos e indurados. Se trasfundieron $600 \mathrm{~mL}$ transquirúrgicos y $300 \mathrm{~mL}$ posquirúrgicos. La paciente tuvo egreso hospitalario sin complicaciones, con antibióticos y analgesia ambulatoria, además de fármacos antitrombóticos.

Después de analizar el espécimen de nefrectomía izquierda y los ganglios linfáticos, el servicio de Patología estableció el diagnóstico de carcinoma de células renales. Tamaño del tumor: 15.5 x $16 \mathrm{~cm}$, con necrosis de $60 \%$, que traspasaba la cápsula renal e infiltraba el tejido adiposo perirrenal; con localización en el polo inferior e invasión linfovascular. El seno renal estaba infiltrado por el tumor. Los ganglios linfáticos, en el tejido cercano al hilio renal, con metástasis a un ganglio. Estadio patológico: pTNM pT3a, pN1, M1 (Figuras 3, 4 y 5). La paciente fue enviada al Instituto Nacional de Cancerología donde se solicitó el estudio de inmunohistoquímica para la determinación de proteína TFE3, que complementó el diagnóstico de carcinoma de células renales con translocación del cromosoma Xp11.2. Actualmente se encuentra en el cuarto ciclo de quimioterapia con pazopanib, con enfermedad diseminada a los pulmones, mesenterio y región cervical.

\section{DISCUSIÓN}

El carcinoma de células renales con translocación del cromosoma Xp11.2 y fusión del gen TFE3 se debe a la fractura del cromosoma; la mayor parte de los genes se ha identificado en la fusión del gen TFE3 y en al menos 6 posibles patrones que inducen su sobreexpresión como: ${ }^{1,6}$ 


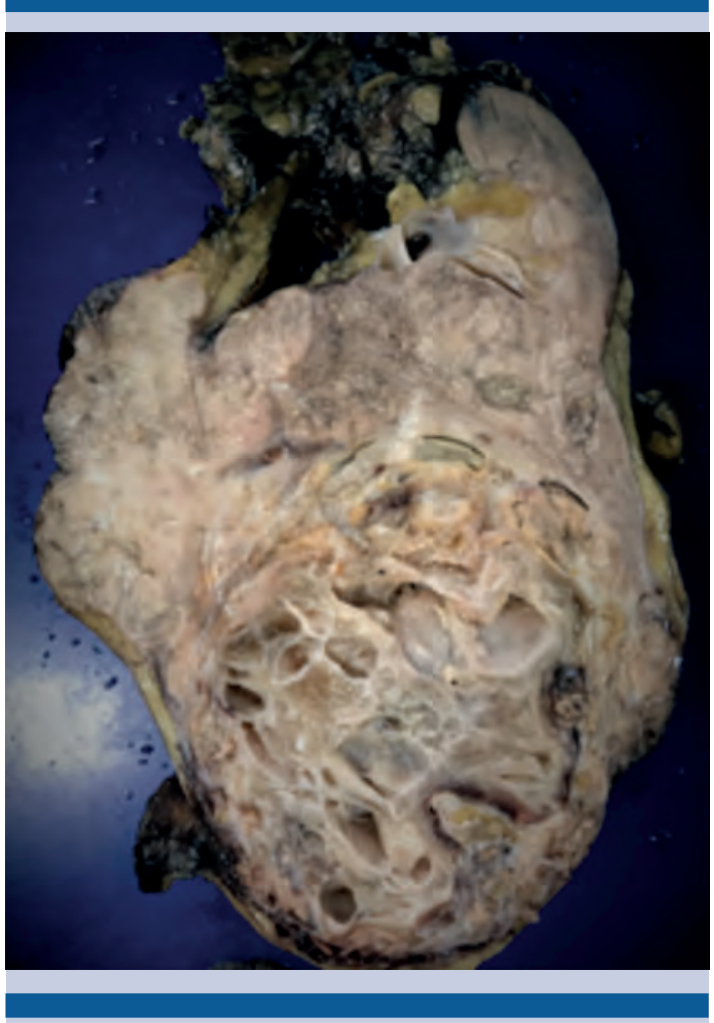

Figura 3. Pieza quirúrgica, compatible con tumor renal en el polo inferior, heterogéneo, de $15 \times 16 \mathrm{~cm}$, con áreas sólidas, quísticas y de necrosis extensa.

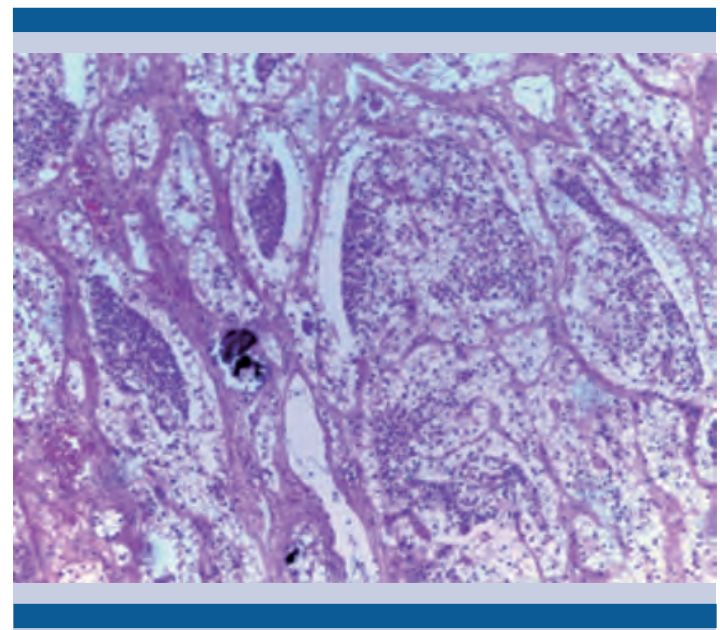

Figura 4. Corte histológico que muestra áreas de aspecto papilar, con pleomorfismo marcado (40x).

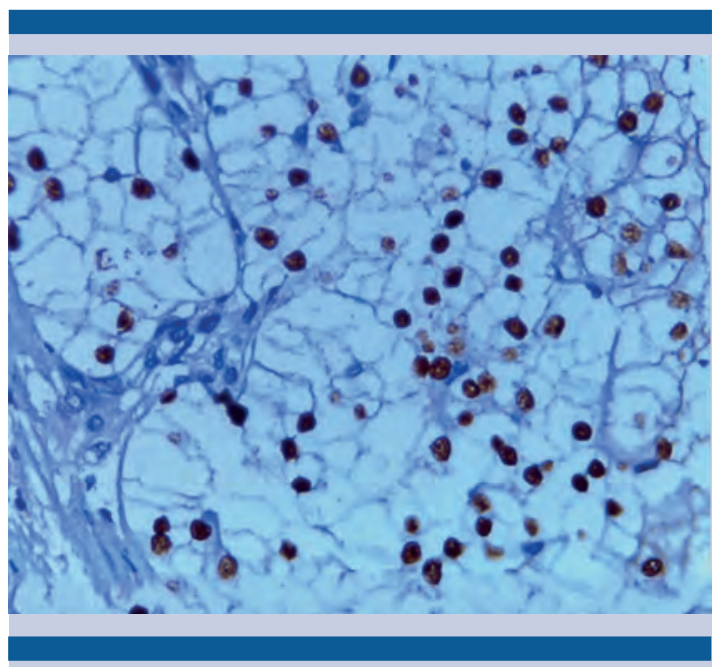

Figura 5. Inmunohistoquímica positiva para TFE3 nuclear, con áreas de patrón bifásico.

PRCC (1q21), PSF (1q34), CLTC (17q23), ASPL (17q25), NONO (Xq12), ASPSCR1, SFPQ, LUC7L3, KHSRP y PARP14. Hasta la fecha, los genes con mayor sobreexpresión son ASPSCR1 y PRCC. ${ }^{1}$ Algunos estudios reportan que ASPSRC1TFE3 suele aparecer en etapas avanzadas del tumor, lo que puede ser útil en la repercusión del pronóstico. ${ }^{9}$ Investigaciones recientes demuestran que el comportamiento biológico del tumor con translocación Xp11.2 en pacientes con cáncer renal puede diferir según el subgrupo en el que se encuentre, además de los patrones de sobreexpresión del gen TFE3. ${ }^{6-8}$

El carcinoma de células renales con translocación Xp11.2 difícilmente crece como un tumor primario, más allá de los límites normales del riñón; sin embargo, en el caso aquí reportado, al momento de establecer el diagnóstico se encontró una tumoración que traspasaba la cápsula renal, con infiltración del tejido perirrenal. Casi $35 \%$ de los carcinomas renales en niños se asocian con la translocación del gen TFE3. 
Este tumor es agresivo en pacientes de edad avanzada, provoca metástasis a distancia y consecuencias clínicas desfavorables, debido a su detección en estadios avanzados de la enfermedad, datos que coinciden con las características de nuestra paciente (64 años de edad, con tumoración mayor de $15 \mathrm{~cm}$, diseminación ganglionar perihiliar bilateral, adyacentes a la arteria aorta, que desplazaban en sentido anterior a la vena cava). La incidencia de la translocación Xp11.2 asociada con carcinoma renal se ha estimado en $1.5 \%$ de los tumores renales de pacientes adultos. Es probable que la afectación en mujeres se deba a que poseen dos cromosomas $\mathrm{X}$, mientras que los hombres solo uno; de ahí la mayor incidencia en la translocación del cromosoma $\mathrm{X}$ o, quizá, mayor posibilidad de manifestación. ${ }^{1,4}$

\section{Histopatología}

El tumor renal de translocación Xp11.2 suele tener estructura papilar o alveolar y está compuesto por células con citoplasma eosinofílico voluminoso y claro. ${ }^{6,7}$ También se han encontrado células claras, núcleo vesicular y figuras psamomatosas como características histopatológicas comunes, incluso tumores de células epiteliales perivasculares o melanoma. ${ }^{9}$ La catepsina k, los marcadores melanocíticos y la proteína TFE3 se expresan frecuentemente en los estudios de inmunohistoquímica. ${ }^{1}$ Desde el punto de vista patológico, se origina de la médula renal, contrario a lo que ocurre en los casos de carcinoma renal de células claras, que se produce en la corteza renal. $7,8,10$

Alguno estudios indican que este tipo de tumores puede subestimarse en pacientes adultos, porque morfológicamente imitan un carcinoma renal de células claras o papilares, (63-68\% de la translocación Xp11.2 en pacientes con carcinoma renal se diagnostican como papilar con células eosinofílicas). ${ }^{6,11}$ En relación con esto, existen casos excepcionales con carcinoma de células renales con translocación del cromosoma Xp11.2 y una tumoración contralateral con patrón de células claras que enmascaran una neoplasia de la otra; por tanto, debe considerarse efectuar las pruebas necesarias para establecer el diagnóstico certero. ${ }^{12-14}$

\section{Diagnóstico}

En la actualidad el estudio de referencia para identificar la translocación del cromosoma Xp11.2 asociada con carcinoma de células renales es la hibridación con fluorescencia in situ (FISH), que expresa la fusión del gen TFE3. ${ }^{1,9}$ Las células tumorales nucleares expresan fuertemente la proteína TFE3; por tanto, se considera un marcador inmunitario con alta sensibilidad (97.5\%) y especificidad (99.6\%) para establecer el diagnóstico de estos tumores. Así, pues, representa una técnica más práctica, comparada con FISH, en instituciones como la nuestra. ${ }^{3,10,15}$ Este anticuerpo reconoce la porción C terminal de la proteína TFE3 y está disponible para distinguir la translocación de Xp11 de otros subtipos de carcinoma renal. También se ha demostrado que la proteína TFE3 se expresa en otros tejidos, pero es regulada de forma estrecha, por lo que tiene concentraciones indetectables en la inmunotinción de tejidos sanos. ${ }^{3}$ Aunque la hibridación con fluorescencia in situ es ampliamente utilizada en diferentes centros hospitalarios, su valor predictivo positivo es bajo; por lo que debe complementarse con otros estudios para aumentar la sensibilidad y especificidad. ${ }^{16}$

Otros métodos para establecer el diagnóstico incluyen el cariotipo y PCR, con tejido fresco o congelado, pero no se encuentran disponibles en la mayor parte de los laboratorios clínicos. ${ }^{3}$

El carcinoma de células renales con translocación del cromosoma Xp11.2 suele ser un hallazgo en estudios radiológicos en pacientes asintomáticos; sin embargo, se han reportado 
casos con manifestaciones como: dolor de espalda, dolor abdominal, hematuria y anemia. El tumor se identifica en etapas avanzadas de la enfermedad, con evolución agresiva en sujetos adultos. La paciente de este estudio tuvo dolor abdominal de dos meses de evolución y masa palpable de grandes dimensiones; además de la edad a la manifestación y etapa clínica avanzada, su pronóstico es malo. ${ }^{1,6}$

El estudio de imagen puede evidenciar una masa fuerte o medianamente hiperdensa, con patrón realzado y límite notorio (signo encapsulado) en la tomografía. ${ }^{10}$ Nuestra paciente se encontraba en etapa clínica avanzada, con una tumoración de gran tamaño que traspasaba la cápsula renal e infiltraba el tejido adiposo perirrenal; por lo tanto, el estudio de imagen mostró una masa de bordes irregulares, contenido heterogéneo y calcificaciones. Algunos reportes indican que las calcificaciones pueden simular alteraciones renales benignas y al establecer el diagnóstico final se trata de carcinoma de células renales con translocación Xp11.2, por lo que debe efectuarse el estudio completo de los pacientes, con la finalidad de implementar el tratamiento adecuado y oportuno. ${ }^{17}$

Existen investigaciones que muestran fuerte correlación con la diseminación a nódulos linfáticos regionales al momento del diagnóstico $(26.5 \%)$, pero tal asociación fue reportada, también, posterior al procedimiento quirúrgico. ${ }^{6}$ Este tipo de diseminación representa mal pronóstico en pacientes adultos, no así en los jóvenes. ${ }^{4}$ En el caso aquí reportado se encontraron dos ganglios linfáticos en el tejido cercano al hilio renal, con metástasis en un ganglio linfático y un ganglio con hiperplasia folicular reactiva, que confieren un mal pronóstico.

La extensión extracapsular se asocia clínica y patológicamente con tumores pequeños (menores de $4 \mathrm{~cm}),{ }^{16}$ incluso diversos estudios sugieren que las masas solitarias con pequeño diámetro (menores de $7 \mathrm{~cm}$ ) y límites claros son factores de buen pronóstico; sin embargo, estos datos aún se discuten. ${ }^{4}$ El estadio avanzado y la trombosis del tumor en la vena cava inferior son los factores más significativos para predecir un mal pronóstico. Se han reportado casos excepcionales con metástasis a huesos u ovarios..$^{8,18}$

\section{Tratamiento}

El tratamiento de pacientes con carcinoma de células renales con translocación Xp11.2 depende de la edad en que se manifiesta y la etapa clínica, pero casi siempre se indica disección regional de nódulos linfáticos o terapia coadyuvante. ${ }^{6}$

La nefrectomía radical es el procedimiento de elección. ${ }^{4}$ La disección de ganglios linfáticos en conjunto con nefrectomía radical puede tener potenciales beneficios para pacientes con carcinoma renal de translocación Xp11. 3,19 En nuestra paciente se efectuó nefrectomía radical izquierda y linfadenectomía.

Entre las modalidades farmacológicas se encuentran: quimioterapia, inmunoterapia y terapia molecular blanco. La quimioterapia tiene función limitada como tratamiento, pues supone baja sensibilidad y toxicidad significativa. En los últimos años se ha explorado que el cáncer renal origina una respuesta inmunitaria; sin embargo, no ha logrado demostrar ventajas en la supervivencia. Las dosis bajas de interleucina-2 e interferón- $\alpha$ representan el único tratamiento efectivo en pacientes menores de 60 años de edad con tumores de bajo grado (algunos casos sugieren toxicidad media).

La terapia blanco ha demostrado mayor sensibilidad y periodos libres de enfermedad más largos que la inmunoterapia en pacientes con enfermedad localmente avanzada y metástasis. En sujetos jóvenes es limitada su indicación, debido a la 
alta toxicidad durante el desarrollo y porque tienen mejor pronóstico que los adultos. ${ }^{16}$

Algunos reportes sugieren que la terapia con factor de crecimiento vascular endotelial es tan efectiva como en otros tejidos de sujetos con carcinoma renal; no obstante, la eficacia de estos fármacos aún no se comprueba en pacientes con la variante de translocación Xp11. ${ }^{3}$

En la actualidad la terapia con inhibidores del receptor de tirosina-cinasa (sunitinib, sorafenib y everolimus), prescrita en paciente con nódulos linfáticos o metástasis a distancia, ha reportado una tasa de supervivencia libre de enfermedad de 8.2 meses, comparada con la monoterapia de citocinas que solo brinda dos meses de vida. ${ }^{4}$ Incluso se han identificado vías que regulan la transición epitelial mesenquimal del receptor de tirosina-cinasa, que activan las vías de cinasa fosfatidilinositol-3 y mTOR. Considerando lo anterior, se sugiere que los inhibidores de mTOR también pueden ser efectivos en este tipo de tumores. ${ }^{5}$ Actualmente, la paciente de este estudio recibe el cuarto ciclo con pazopanib, con el que se ha observado buena reacción.

Durante el seguimiento de pacientes con carcinoma de células renales con translocación Xp11.2 debe efectuarse examen físico, análisis de laboratorio, tomografía de tórax cada 6 meses los primeros tres años a partir del diagnóstico y después anualmente por 10 años. La tomografía de abdomen debe solicitarse cada 6 meses durante los primeros dos años, anualmente entre los 2-5 años y después cada 2 años por 10 años. ${ }^{16}$

\section{CONCLUSIÓN}

Hasta la fecha no existen reportes adicionales de pacientes con carcinoma de células renales con translocación del cromosoma Xp11.2 en México, la mayor parte se han descrito en España y Venezuela. Aunque la paciente de este estudio tiene diversos factores de mal pronóstico: 64 años de edad, sexo femenino, etapa avanzada, estadio pT3a pN1, diseminación a ganglios linfáticos, tumoración mayor a $10 \mathrm{~cm}$ y crecimiento extracapsular con infiltración al tejido perirrenal, el seguimiento y la supervivencia se han prolongado, incluso, 12 meses.

\section{REFERENCIAS}

1. Al-Maghrabi AJ, et al. Uncommon localization of extrarenal xp11.2 translocation-associated renal cell carcinoma (RCC): Case report. Appl Immunohistochem Mol Morphol 2017. DOI: $10.1097 /$ PAI.0000000000000576

2. Lim B, et al. Clinicopathological features of Xp11.2 translocation renal cell carcinoma. Korean J Urol 2015;56(3):212217. DOI: 10.4111/kju.2015.56.3.212

3. Hirobe $M$, et al. Clinicopathological characteristics of Xp11.2 translocation renal cell carcinoma in adolescents and adults: Diagnosis using immunostaining of transcription factor E3 and fluorescence in situ hybridization analysis. Int J Urol 2016;23(2):140-145. DOI: 10.1111/iju.13007

4. Liu N, et al. Renal cell carcinoma associated with Xp11.2 translocation /TFE3 gene fusions: clinical features, treatments and prognosis. Plos One 2016;11(11):e0166897. DOI: 10.1371/journal.pone.0166897

5. Kakoki K, et al. Long-term treatment with sequential molecular targeted therapy for Xp11.2 translocation renal cell carcinoma: A case report and review of the literature. Clin Genitourin Cancer 2017;15(3):e503-e506. DOI: 10.1016/j. clgc.2016.12.026

6. Choo MS, et al. Clinicopathologic characteristics and prognosis of Xp11.2 translocation renal cell carcinoma: multicenter, propensity score matching analysis. Clin Genitourin Cancer 2017;15(5):e819-e825. DOI: 10.1016/j. clgc.2017.04.015

7. Manucha V, et al. Cyto-histological correlation of Xp11.2 translocation/TFE3 gene fusion associated renal cell carcinoma: Report of a case with review of literature. Diagn Cytopathol 2018;46(3):267-270. DOI: 10.1002/dc.23839

8. Liu J, et al. Xp11.2 translocation renal cell carcinoma with multiple bone metastases: A case report. Oncol Letters 2016;11(3):2233-2236. DOI: 10.3892/ol.2016.4211

9. Classe $\mathrm{M}$, et al. Incidence, clinicopathologic features and fusion transcript of translocation renal cell carcinomas. Histopathology 2017;70(7):1089-1097. DOI: 10.1111/ his.13167

10. Chen $X$, et al. Renal cell carcinoma associated with Xp11.2 translocation/TFE gene fusion: imaging findings in 21 patients. Eur Radiol 2017;27(2):543-552. DOI: 10.1007/ s00330-016-4421-4

11. Ahluwalia $P$, et al. Renal cell carcinoma associated with Xp 11.2 Translocation/TFE3 gene fusion: A rare case 
report with review of the literature. Hindawi Publishing Corporation. Case Rep Urol 2013;2013:810590. DOI: $10.1155 / 2013 / 810590$

12. D'Antonio A, et al. Unsuspected Xp11 translocation renal neoplasm associated with contralateral clear cell carcinoma. Int J Surg Pathol 2016;24(3):248-252. DOI: 10.1177/1066896915597753

13. Asaki HE, et al. Xp11.2 translocation tumor: A rare cause of gross hematuria. JAAPA 2014;27(2):24-27. DOI: 10.1097/01.JAA.0000442700.87975.0b

14. Parihar A, et al. Xp11 translocation renal cell carcinoma morphologically mimicking clear cel-papillary renal cell carcinoma in an adult patient: report of a case expanding the morphologic spectrum of xp11 translocation renal cell carcinomas. Int J Surg Pathol 2015;23(3):243-7. DOI: $10.1177 / 1066896914562280$

15. Nishimura K, et al. A case of metastatic Xp11.2 translocation renal cell carcinoma successfully managed by cytoreductive nephrectomy followed by axitinib therapy. Mol Clin Oncol 2017;6(3):362-364. DOI: 10.3892/mco.2017.1142
16. Wang $Z$, et al. Postoperative recurrence of adult renal cell carcinoma associated with Xp11.2 translocation/TFE3 gene fusion. J Int Med Res 2017;45(4):1287-1296. DOI: 10.1177/0300060517711358

17. Liang $\mathrm{W}$, et al. Xp11.2 translocation renal cell carcinoma with egg-shell calcification mimicking a benign renal tumour: A case report. Oncol Letters 2015;10(5):3191-3194. DOI: 10.3892/ol.2015.3718

18. Quiñonez-Urrego $E$, et al. Carcinoma renal asociado con translocacion Xp11. Analisis de dos casos del adulto, uno de ellos con presentación primaria como metástasis ovárica. Patol Rev Latinoamer 2013;51(4):239-243. Dirección URL: $<$ https://www.researchgate.net/publication/259604844 Carcinoma_renal_asociado_con_translocacion_Xp11_ Analisis_de_dos_casos_del_adulto_uno_de_ellos_con_ presentacion_primaria_como_metastasis_ovarica>.

19. Akhavein A, et al. Xp11 translocation renal cell carcinoma: unusual variant masquerading as upper tract urothelial cell carcinoma. Urol Case Rep 2014;2(3):75-77. DOI: 10.1016/j. eucr.2014.02.003

\section{AVISO IMPORTANTE}

La Revista Mexicana de Urología se convierte en una publicación solo digital, con todas las ventajas que los medios y dispositivos electrónicos ofrecen. Usted podrá revisar la información mediante el sitio web (www.revistamexicanadeurologia.org.mx) o descargando la app para Android o iPhone.

Para consultar el texto completo de los artículos deberá registrarse por una sola vez con su correo electrónico, crear una contraseña, indicar su nombre, apellidos y especialidad.

Esta información es indispensable para saber qué consulta y cuáles son sus intereses, y poder en el futuro inmediato satisfacer sus necesidades de información. 